\title{
Size-specific follicle selection improves mouse oocyte reproductive outcomes
}

\author{
Shuo Xiao ${ }^{1,2}$, Francesca E Duncan ${ }^{3}$, Lu Bai ${ }^{1,4}$, Catherine T Nguyen ${ }^{1,4}$, Lonnie D Shea ${ }^{5}$ \\ and Teresa K Woodruff ${ }^{1,2}$ \\ ${ }^{1}$ Department of Obstetrics and Gynecology, Feinberg School of Medicine, Northwestern University, Chicago, \\ Illinois 60611, USA, ${ }^{2}$ Center for Reproductive Science, Northwestern University, Evanston, Illinois 60208, USA, \\ ${ }^{3}$ Department of Anatomy and Cell Biology, University of Kansas Medical Center, Kansas City, Kansas 66160, USA, \\ ${ }^{4}$ Master of Biotechnology Program, Northwestern University, Evanston, Illinois 60628, USA and ${ }^{5}$ Department of \\ Biomedical Engineering, College of Engineering and Medical School, University of Michigan, \\ Ann Arbor, Michigan 48109, USA
}

Correspondence should be addressed to T K Woodruff; Email: tkw@northwestern.edu

\begin{abstract}
Encapsulated in vitro follicle growth (eIVFG) has great potential to provide an additional fertility preservation option for young women and girls with cancer or other reproductive health threatening diseases. Currently, follicles are cultured for a defined period of time and analyzed as a cohort. However, follicle growth is not synchronous, and culturing follicles for insufficient or excessive times can result in compromised gamete quality. Our objective is to determine whether the selection of follicles based on size, rather than absolute culture time, better predict follicle maturity and oocyte quality. Multilayer secondary mouse follicles were isolated and encapsulated in $\mathbf{0 . 2 5} \%$ alginate. Follicles were cultured individually either for defined time periods or up to specific follicle diameter ranges, at which point several reproductive endpoints were analyzed. The metaphase II (MII) percentage after oocyte maturation on day 6 was the highest $(85 \%)$ when follicles were cultured for specific days. However, if follicles were cultured to a terminal diameter of 300-350 $\mu \mathrm{m}$ irrespective of absolute time in culture, 93\% of the oocytes reached MII. More than 90\% of MII oocytes matured from follicles with diameters of 300-350 $\mu \mathrm{m}$ showed normal spindle morphology and chromosome alignment, 85\% of oocytes showed two pronuclei after IVF, $81 \%$ developed into the two-cell embryo stage and $38 \%$ developed to the blastocyst stage, all significantly higher than the percentages in the other follicle size groups. Our study demonstrates that size-specific follicle selection can be used as a non-invasive marker to identify high-quality oocytes and improve reproductive outcomes during eIVFG.

Reproduction (2015) 150 183-192
\end{abstract}

\section{Introduction}

Young cancer patients undergoing chemotherapy and radiation treatments may experience threatened fertility as an unintended consequence of these life-saving medical interventions (Jeruss \& Woodruff 2009). This issue has been at the forefront of reproductive research activities over the past 10 years and many new technologies have been developed to help these patients preserve their fertility (De Vos et al. 2014). In addition to cancer, there are also non-malignant diseases and conditions, as well as their treatments, that can negatively affect reproductive function (Hirshfeld-Cytron et al. 2011, Purcell \& Moley 2011). In addition to fertility concerns, loss of endocrine support of hormonally responsive tissues can cause a cascade of medical and quality-of-life problems and must be addressed as part of the initial comprehensive plan of care for young women.
To address the fertility needs of young women and girls with any fertility-threatening condition or treatment, we have developed an alginate hydrogel-based encapsulation system that supports the growth, development and maturation of gamete-containing follicles outside the context of the ovary (Xu etal. 2006a). This culture method maintains follicle architecture and the spatial relationship of the oocyte and its supporting somatic cells. This method is significant because it provides a potential alternative to ovarian tissue transplantation for preserving fertility and does not have the inherent risk of reintroducing cancer cells because follicles develop completely in vitro (Woodruff 2007). Encapsulated in vitro follicle growth (elVFG) has successfully resulted in live births in mice (Xu et al. 2006a), in addition to follicle growth, oocyte development and preimplantation embryo development in other large mammalian species (Xu et al. 2009a,b, 2010, 2011a, Songsasen et al. 2011). 
Thus, elVFG is one of several other systems that has been successful in supporting the in vitro growth and development of ovarian follicles (Smitz et al. 2010, Telfer \& McLaughlin 2012). However, despite the promise of this technology, there is significant room for improvement, as the efficiency of the technique in terms of IVF success and live birth outcomes remains low in the mouse (Xu et al. 2006a). Moreover, there are unique challenges in translating this work from the mouse to primates because of distinct species differences in follicle growth patterns and requirements (Xu et al. 2011a).

Hydrogel-based methods of elVFG permit the growth and development of follicles and oocytes in vitro, followed by hormone-induced oocyte maturation to stimulate coordinated ovulation and meiotic resumption in the oocyte (Xu et al. 2006a). During elVFG, follicles are typically isolated and cultured for a defined period of time and analyzed as a cohort before performing the oocyte maturation. However, follicle growth in culture is not synchronous, which means that at any given point there may only be a fraction of follicles that are ready to mature. We hypothesize that this asynchrony combined with our inability to select follicles that contain a fullygrown oocyte may contribute to the reduced efficiency of eIVFG. To improve the elVFG system and produce fully mature, high-quality oocytes that are competent to be fertilized and produce viable embryos, it is critical to define the point at which cultured follicles are fullygrown and oocytes have achieved full developmental potential. The primary objective of the present study was to monitor mouse follicles individually to determine whether size-specific follicle selection, rather than absolute culture time, can be used as a non-invasive marker to identify follicles during elVFG to ultimately improve reproductive outcomes using this technique. This is particularly important for human elVFG, where follicles take longer periods of time ( $>30$ days) to reach maturity (Xu et al. 2009a).

\section{Materials and methods}

\section{Animals}

Immature follicles were isolated from ovaries harvested from 16-day-old CD1 female mice. For control for IVF, mature eggs were collected from the oviducts of hyper-stimulated adult CD1 female mice. Sperm were collected from the cauda epipdidymi of proven CD1 male breeders. All mice were housed in polypropylene cages and provided food and water ad libitum. Animals were kept on a $12 \mathrm{~h}$ light: $12 \mathrm{~h}$ darkness cycle $(0700-1900 \mathrm{~h})$ at $23 \pm 1{ }^{\circ} \mathrm{C}$ with $30-50 \%$ relative humidity. Animals were fed Teklad Global irradiated 2919 or 2916 chow (Madison, WI, USA), which does not contain soybean or alfalfa meal to minimize exposure to phytoestrogens. All methods used in this study were approved by the Northwestern University Institutional Animal Care and Use Committee (IACUC) and correspond to the National Institutes of Health guidelines and public law.

\section{Follicle isolation, encapsulation and culture}

Multilayer secondary follicles $(150-180 \mu \mathrm{m}$, type 5b) were isolated from 16-day-old CD1 female mice as previously described (Xu et al. 2006a). Only follicles that displayed intact morphology were selected for encapsulation and culture. Follicles were placed in maintenance media containing 50\% minimal essential medium ( $\alpha$-MEM Glutamax) and $50 \%$ Nutrient Mixture (F-12 with Glutamax) with $1 \%$ fetal bovine serum (FBS, Life Technologies) for $2 \mathrm{~h}$ before encapsulation. Selected follicles were then encapsulated individually in $0.25 \%$ alginate (NovaMatrix, Sandvika, Norway) as previously described (Xu et al. 2006a,b). Alginate beads were placed in 96-well plates, with each well containing $100 \mu$ l growth media (50\% $\alpha$-MEM Glutamax and 50\% F-12 Glutamax supplemented with $3 \mathrm{mg} / \mathrm{ml}$ BSA (Sigma-Aldrich), $10 \mathrm{mlU} / \mathrm{ml}$ recombinant follicle-stimulating hormone ( $\mathrm{rFSH}$; from A F Parlow, National Hormone and Peptide Program, National Institute of Diabetes and Digestive and Kidney Diseases, Bethesda, MD, USA), $1 \mathrm{mg} / \mathrm{ml}$ bovine fetuin (Sigma-Aldrich), $5 \mu \mathrm{g} / \mathrm{ml}$ insulin, $5 \mu \mathrm{g} / \mathrm{ml}$ transferrin and $5 \mu \mathrm{g} / \mathrm{ml}$ selenium (Sigma-Aldrich)). For all experiments, follicles were maintained at $37^{\circ} \mathrm{C}$. Encapsulated follicles were cultured at $37{ }^{\circ} \mathrm{C}$ in $5 \% \mathrm{CO}_{2}$ in air for specific periods of time $(2,4,6,8$ and 10 days $)$ or until reaching specific follicle diameters of 200-250, 250-300, 300-350 and $350-400 \mu \mathrm{m}$. Half of the growth media $(50 \mu \mathrm{l})$ was replaced every other day. Follicles were imaged at each media change using an inverted Leica DM IRB microscope with $4 \times$ and $20 \times$ objectives (Leica Microsystems, Buffalo Grove, IL, USA). Follicle growth curves were obtained by plotting the average follicle diameter, which was calculated by averaging two perpendicular measurements from basement membrane to basement membrane of each follicle in ImageJ Software (National Institutes of Health, Bethesda, MD, USA). Follicles were considered dead if they had unhealthy-appearing oocytes and/or granulosa cells, or if the integrity of the oocyte and somatic cell interface was visibly compromised. Dead and unhealthy follicles were removed from the culture, and only surviving follicles were included in the analysis. The follicle survival rate is more than 90 and $<10 \%$ of follicles showed unhealthy follicle morphology and degenerated oocyte during the culture.

\section{Analysis of oocyte chromatin configuration}

Follicles cultured for specific periods of time were removed from alginate beads using $10 \mathrm{IU} / \mathrm{ml}$ alginate lyase from Flavobacterium multivorum (Sigma-Aldrich). Oocytes were mechanically isolated from follicles cultured for specific days or when follicles reached specific diameters. Oocytes were then immediately incubated in L15 media with $0.1 \%$ milrinone (Sigma-Aldrich) and $1 \mu \mathrm{g} / \mathrm{ml}$ Hoechst 33342 (Sigma-Aldrich) for $15 \mathrm{~min}$, and immediately analyzed by confocal microscopy (Leica Microsystems). During oocyte development and maturation, oocyte chromatin can be classified by three types of configurations (Zuccotti et al. 1998, Bouniol-Baly et al. 1999). Here we use the following criteria to assign oocytes to these three classes: first, the non-surrounded nucleolus (NSN) was characterized by a cloud of euchromatin occupying the entire 
nucleoplasm, which contains some chromatin foci. The second configuration is the surrounded nucleolus ( $\mathrm{SN})$, which was characterized by condensed heterochromatin surrounding the nucleolus and a nucleoplasm with minimal patches of euchromatin. Finally, the intermediate stage (I) configuration is between NSN and SN and was characterized by a partial rim of perinucleolar chromatin around the nucleolus.

\section{Oocyte maturation, superovulation, fertilization and embryo culture}

Oocyte maturation was performed on specific days of follicle culture or when the follicles reached a specific diameter ranges. Follicles were removed from alginate beads and incubated for $16 \mathrm{~h}$ at $37{ }^{\circ} \mathrm{C}$ in $5 \% \mathrm{CO}_{2}$ in air in maturation media $(\alpha$-MEM with $10 \%$ FBS, $1.5 \mathrm{IU} / \mathrm{ml}$ human chorionic gonadotrophin (hCG), $10 \mathrm{ng} / \mathrm{ml}$ epidermal growth factor (BD Biosciences, Franklin Lakes, NJ, USA) and $10 \mathrm{mIU} / \mathrm{ml} \mathrm{rFSH}$ ). Oocytes were then denuded from the surrounding cumulus cells using $0.3 \%$ hyaluronidase (Sigma-Aldrich). Oocytes were considered to be arrested at prophase I in the germinal vesicle (GV) stage if the nucleus was intact, but were considered to have undergone GV breakdown (GVBD) if the nucleus was not visible. If a polar body was present in the perivitelline space, the oocytes were classified as metaphase II (MII). Fragmented or shrunken oocytes were classified as degenerated (D).

For IVF, mature motile sperm were collected from the cauda epididymis of proven CD1 male breeders and capacitated in human tubal fluid (HTF) medium with $4 \mathrm{mg} / \mathrm{ml}$ BSA for $1.5 \mathrm{~h}$ (Kito et al. 2004). MIl oocytes from follicles with different diameter ranges were collected for oocyte maturation, with follicle culture time periods ranging from 2 to 10 days. At least 20-40 oocytes were collected for each follicle size group. To eliminate the effect of zona pellucida (ZP) hardening on insemination, the ZP of the denuded MII oocytes was removed by incubating oocytes in acidic Tyrodes solution (SigmaAldrich) for 10-15 s. ZP-free oocytes were washed three times in HTF medium and combined with $50000 \mathrm{sperm} / \mathrm{ml}$ for $4 \mathrm{~h}$ to increase fertilization rates and reduce the incidence of polyspermy (Evans et al. 1995, McAvey et al. 2002). Fertilized oocytes were identified by the presence of two pronuclei (2PN). Oocytes were then washed in fresh KSOM (EMD Millipore, Billerica, MA, USA) to remove bound sperm and cultured individually in $50 \mu \mathrm{l} \mathrm{KSOM}$ drops until the embryos developed to the blastocyst stage. Superovulated MII oocytes were used as an in vivo control. Superovulation was performed by $5 \mathrm{IU}$ of pregnant mare's serum gonadotrophin (PMSG; Sigma-Aldrich) i.p. injection into the adult female mice, which was followed by $5 \mathrm{IU}$ hCG injection $48 \mathrm{~h}$ after the PMSG injection. MII oocytes were collected from the ampulla $14 \mathrm{~h}$ after the hCG injection, and the ZP of MII oocytes was removed before performing IVF.

\section{Histology}

Follicles cultured in vitro were removed from the alginate beads and fixed for $3 \mathrm{~h}$ at $4{ }^{\circ} \mathrm{C}$ in $3.8 \%$ paraformaldehyde in $1 \times$ PBS. Follicles were dehydrated in ascending concentrations of ethanol (50-100\%) and re-encapsulated in 1\% alginate hydrogel before being embedded in paraffin using an automated tissue processor. Serial $5 \mu \mathrm{m}$ sections were cut and stained with hematoxylin and eosin to identify the follicle diameter when antral cavity and cumulus-oocyte complex (COC) were formed during elVFG.

\section{Immunofluorescence}

For spindle morphology and chromosome alignment analysis, gametes obtained following oocyte maturation were fixed in $3.8 \%$ paraformaldehyde containing $0.1 \%$ Triton X-100 (SigmaAldrich) for $1 \mathrm{~h}$ at $37{ }^{\circ} \mathrm{C}$. Oocytes were washed three times in blocking solution with $1 \times$ PBS containing $0.3 \%$ BSA and $0.01 \%$ Tween-20, incubated overnight in a 1:50 dilution of mouse anti- $\alpha$-tubulin (Cell Signaling Technology, Danvers, MA, USA) in blocking solution. Then, oocytes were washed three times with blocking solution, mounted using Vectashield containing DAPI (Vector Laboratories, Burlingame, CA, USA), and analyzed using an EVOS FL AUTO microscope (Life Technologies) in a blinded fashion. Oocytes with barrel-shaped bipolar spindles and well-organized microtubule fibers, along with tightly aligned chromosomes on the metaphase plate, were scored as normal. All other configurations were considered abnormal.

For chromosome cohesion analysis, gametes obtained following superovulation or in vitro oocyte maturation were cultured for $1 \mathrm{~h}$ in CZB containing $100 \mu \mathrm{M}$ monastrol (SigmaAldrich), a kinesin-5 inhibitor, which causes the bipolar MII spindle to collapse into a monoplar spindle and results in the dispersion of chromosomes. Oocytes were then incubated with human CREST autoimmune serum (ImmunoVision, Springdale, AR, USA), which was detected by an Alexa-Fluor 594conjugated goat anti-human secondary antibody (Invitrogen). Oocytes were washed in blocking solution, incubated in a 1:5000 solution of Syto Green nucleic acid stain (Invitrogen) for 10 min, mounted in Vectashield and analyzed as previously described (Duncan et al. 2009).

\section{Statistical analysis}

Follicle size, oocyte size and IVF data were analyzed from three to five independent cultures in which 16-24 follicles were cultured for each experimental group. Data were analyzed using one-way ANOVA, followed by the Tukey range test for significant difference. Categorized data were analyzed by the $\chi^{2}$ test; if significance was observed between groups, then we applied the Fisher's exact test. The significance level was set at $P<0.05$.

\section{Results \\ Follicle size correlated with stage-specific follicle development during eIVFG}

To better understand the relationship between follicle size and follicle development during elVFG, we used the mouse model to define the specific follicle diameters associated with key stage-specific follicle developmental events and other reproductive outcomes. Alginate-based hydrogel encapsulation maintained follicle structure and phenocopied in vivo folliculogenesis from the multilayer 
secondary follicle to the early antral and late antral follicle stages (Fig. 1A). The average follicle diameter increased from $158 \pm 15 \mu \mathrm{m}$ on day 0 to $376 \pm 31 \mu \mathrm{m}$ on day 8 and $390 \pm 29 \mu \mathrm{m}$ on day 10 (Fig. 1B). The average size of the oocyte increased from $60 \pm 3$ to $72 \pm 4 \mu \mathrm{m}$ from days 0 to 8 and up to $74 \pm 4 \mu \mathrm{m}$ on day 10 (Fig. 1B). Our prior studies were limited to non-invasive light microscopy analysis of follicle morphology, which included the development of a central cleared space described as the antrum and the movement of the oocyte to an acentric position. Here we extend these gross/ superficial phenotypes to a more systematic histologic analysis of antral formation and cumulus-oocyte interactions. Using this terminal analysis required removal of follicles from culture on each day. The histology data showed that the antral cavity was present only after follicles reached $257 \pm 19 \mu \mathrm{m}$, with this transition occurring in $8 \%$ of follicles cultured on day $3,75 \%$ of follicles cultured on day 4 and $17 \%$ of follicles on day 5 (Fig. $1 \mathrm{~A}$ and $\mathrm{C}$ ). The oocytes became surrounded by cumulus cells and formed COCs when the follicle diameter reached $308 \pm 26 \mu \mathrm{m}$, with this taking place in $12 \%$ of follicles cultured on day $6,63 \%$ of follicles on day 7 and $25 \%$ of follicles on day 8 (Fig. $1 \mathrm{~A}$ and D). After in vitro oocyte maturation, cumulus cell expansion was observed and meiotic resumption occurred, which resulted in the formation of MII oocytes as evidenced by the first polar body extrusion (Fig. 1A). These results demonstrate that follicle stage-specific development correlates with follicle size during eIVFG.

\section{Follicle size correlates with oocyte chromatin configuration during eIVFG}

During oocyte development and maturation, the oocyte chromatin configuration transits from NSN to SN state (De La Fuente \& Eppig 2001). To determine the relationship between oocyte chromatin configuration changes and the follicle growth in our elVFG system, we examined when the NSN to SN transition occurred. On day 0 and day 2 of elVFG, all oocytes within follicles were in the NSN configuration with the chromatin dispersed throughout the nucleolus (Fig. 2A and B). There were oocytes with both the NSN and $\mathrm{SN}$ chromatin configurations on day 4 (Fig. 2A and B), and the oocytes predominantly had a $\mathrm{SN}$ chromatin configuration after day 6 (Fig. $2 \mathrm{~A}$ and B) $(75 \%$ on day $6,96 \%$ on day 8 and $94 \%$ on day 10 respectively; Fig. 2B). Oocytes that had an NSN chromatin configuration were significantly smaller in diameter compared to oocytes with I and SN chromatin configurations (Fig. 2C). This data is consistent with previous studies demonstrating that the transition in oocyte chromatin configuration correlates with oocyte development (De La Fuente \& Eppig 2001). The average follicle diameter was $181 \pm 26$, $237 \pm 31$ and $330 \pm 64 \mu \mathrm{m}$ for follicles containing oocytes with NSN, intermediate and SN chromatin configurations, respectively (Fig. 2D). These data indicate that oocyte chromatin configuration transitions from NSN to SN state when follicles reach the antral stage during elVFG (Fig. 1), which correlates with oocyte chromatin redistribution in vivo (De La Fuente \& Eppig 2001, Segers et al. 2010). These findings are significant because we are able to define for the first time the precise follicle diameter during elVFG when follicle-enclosed oocytes achieve chromatin configuration in $\mathrm{SN}$ state. This determination is possible with the alginate hydrogel-based elVFG system because the encapsulation maintains the follicle structure, so accurate diameter measurements can be obtained.

\section{Oocyte maturation based on follicle size rather than absolute culture improves oocyte meiosis outcomes}

Oocyte maturation was performed on follicles cultured for specific times or until they reached specific terminal diameter ranges to compare the oocyte meiotic outcomes of these two strategies. The MII percentage of
A

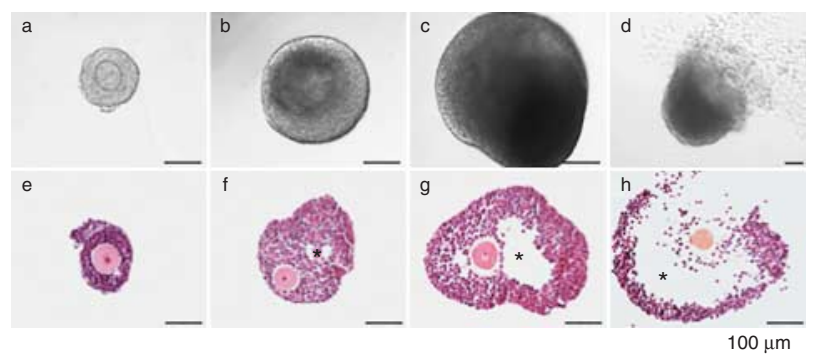

B

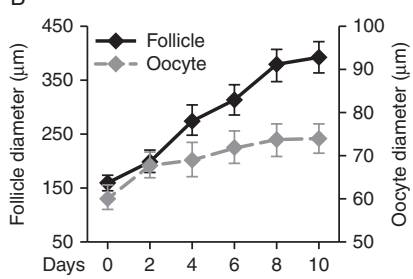

C
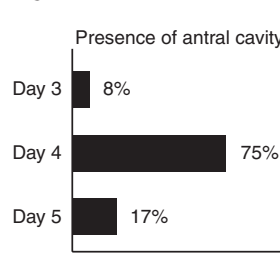

Figure 1 (A) Mouse follicle development in vitro in alginate-based hydrogels. (a, b, c and d) Follicles developed from (a) multilayer secondary follicle to (b) early antral and (c) antral stages during eIVFG. (d) The oocyte extruded the first polar body after oocyte maturation in vitro. Corresponding histology of follicles in different developmental stages during elVFG:

(e) pre-antral stage, (f) early antral stage, (g) antral stage and (h) after oocyte maturation. (B) Follicle and oocyte growth during elVFG from days 0 to 10 . Follicle distribution on different culture days $(C)$ from preantral to antral stage with the presence of antral cavity and (D) cumulus-oocyte complex formation with cumulus cells surrounding the oocyte. Error bars: s.D.; *follicle antrum; scale bar: $100 \mu \mathrm{m} ; n=20-40$ follicles for three replicates. 

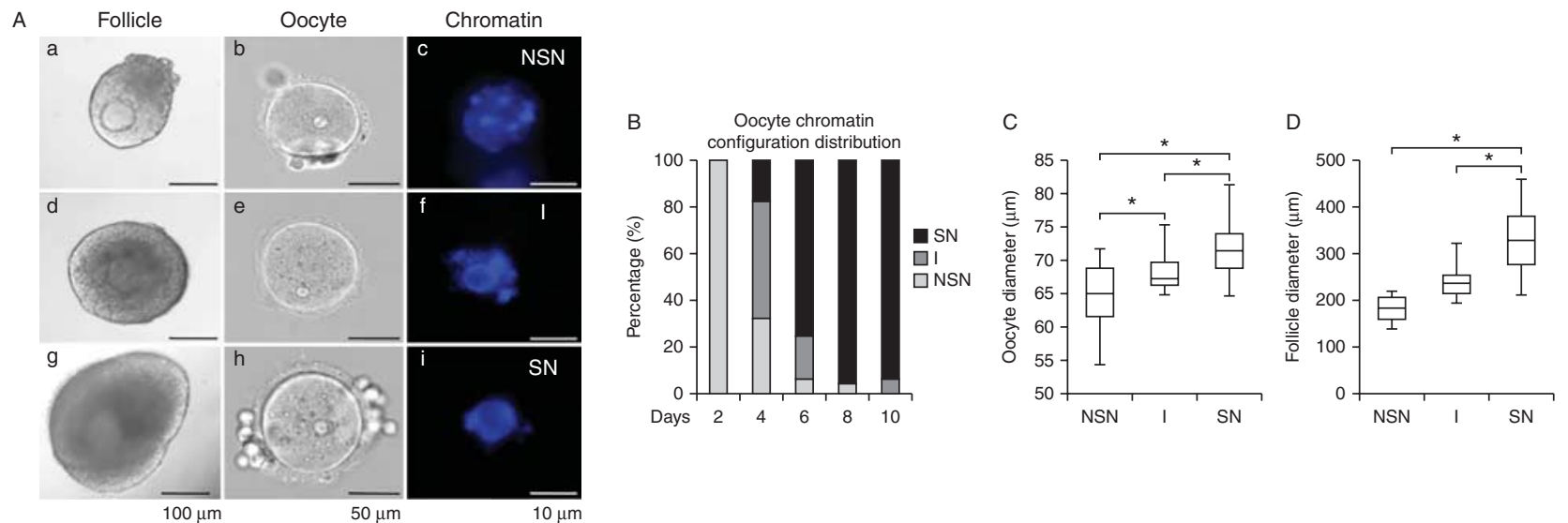

Figure 2 Representative images of follicles and the chromatin configuration within their enclosed oocytes during elVFG (A). (a, b and c) Follicle containing an oocyte with non-surrounded nucleus (NSN); (d, e and f) follicle containing an oocyte with an intermediate stage (I) chromatin configuration and ( $\mathrm{g}$, h and i) follicle containing an oocyte with surrounded nucleus (SN). (B) Chromatin configuration distribution from days 2 to 10 during eIVFG. (C) Oocyte diameters associated with different oocyte chromatin configurations during elVFG. (D) Follicle diameters associated with different oocyte chromatin configurations during elVFG. Scale bars: $100 \mu \mathrm{m}$ for a, d and g; $50 \mu \mathrm{m}$ for b, e and h; $10 \mu \mathrm{m}$ for C, f and i. Error bars: S.D. ${ }^{*} P<0.05$, compared to diameter of follicles with oocyte in I stage in $\mathrm{C}$, and compared to the diameter of follicles with oocyte in $\mathrm{SN}$ stage in $\mathrm{C}$ and $\mathrm{D}$; $n=40-80$ follicles for three replicates.

oocytes from follicles cultured in vitro on day 2 was $25 \%$, indicating that oocytes were not mature and could not support resumption of meiosis (Fig. 3A). The MII percentage on days $4,6,8$ and 10 , were all $>75 \%$, with the MII percentage on day 6 being significantly higher than oocytes matured on days 4,8 and $10(85 \%$ vs $75 \%$, $75 \%$ and $80 \%)$ and with follicle diameter at $289 \pm$ $18 \mu \mathrm{m}$. When oocyte maturation was performed on follicles selected based on size, follicles that ranged from 300 to $350 \mu \mathrm{m}$ showed the highest MII percentage, at 93\% (Fig. 3B). This was significantly higher than the MII percentage for follicles cultured to day $6(85 \%)$ and also higher than the MII percentages for follicles $<250$, 250-300 and $>350 \mu \mathrm{m}$ in diameter (Fig. 3A and B). Follicles that reached diameters of $300-350 \mu \mathrm{m}$ developed over different culture times ranging from days 6 to $9(10 \%$ from day $6,62 \%$ from day $7,24 \%$ from day 8 and $4 \%$ from day 9 respectively). These results indicate that follicle size is a better predictor of oocyte meiotic competence compared to absolute culture time, and follicles with diameter of 300-350 $\mu \mathrm{m}$ showed the best oocyte meiotic competence. Therefore subsequent studies were focused on how follicle size correlated with oocyte reproductive outcomes.

To define the precise follicle diameters at which cultured follicles are fully meiotic competent, follicles were categorized by different meiotic outcomes after oocyte maturation. Oocytes that remained arrested at the GV stage and did not resume meiosis came from follicles that had an average diameter at $173 \pm 59 \mu \mathrm{m}$, while the oocytes that had undergone GVBD were from follicles with a diameter of $280 \pm 12 \mu \mathrm{m}$ (Fig. 3C). Oocytes that were able to reach MII had an average follicle diameter of $305 \pm 67 \mu \mathrm{m}$ (Fig. 3C). The diameters of follicles that produced MII oocytes were significantly larger than those that produced oocytes that failed to resume meiosis (Fig. 3C). Interestingly, degenerated oocytes tended to be observed in follicles with the largest average diameter (367 $\pm 34 \mu \mathrm{m} ;$ Fig. 3C). Based on these data, we conclude that the oocyte maturation strategy based on follicle size is highly correlated to oocyte meiotic outcomes and could therefore have predictive value.

\section{Follicle size correlates with gamete spindle morphology and chromosome alignment}

To study the quality of the mature gametes obtained following elVFG from follicles of different terminal diameters, oocytes that had progressed to MII after oocyte maturation were analyzed for spindle integrity and chromosome alignment, two critical markers of oocyte developmental competence (Moon et al. 2003, Li et al. 2014). Ninety percent of oocytes from follicles cultured to a diameter of 300-350 $\mu \mathrm{m}$ had barrel-shaped bipolar spindles and tightly aligned chromosomes on the metaphase plate. This morphology was comparable to what was observed in MII oocytes produced by superovulation in vivo (Fig. 4A, B, C and G). In contrast, a significantly higher percentage of MIl oocytes from follicles cultured to diameters of $<250$ or $>350 \mu \mathrm{m}$ exhibited abnormal spindle morphology and/or chromosomal misalignment. These oocytes lacked barrel-shaped spindles and their chromosomes were dispersed instead of being tightly aligned on the metaphase plate (Fig. 4D, E, F and G; 35 and $41 \%$ of oocytes respectively). Follicles with diameters between 250 and $300 \mu \mathrm{m}$ produced MII oocytes that had an increased incidence of spindle abnormalities (15\%), but there was no significant difference compared to MII oocytes from 300 to $350 \mu \mathrm{m}$ follicles (Fig. 4G). 


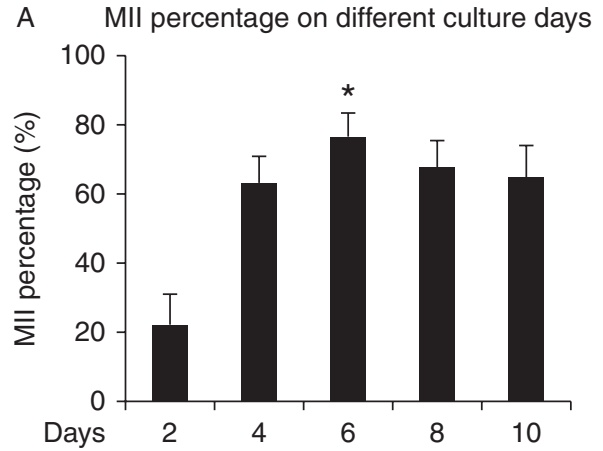

B MII percentage on different follicles diameter ranges

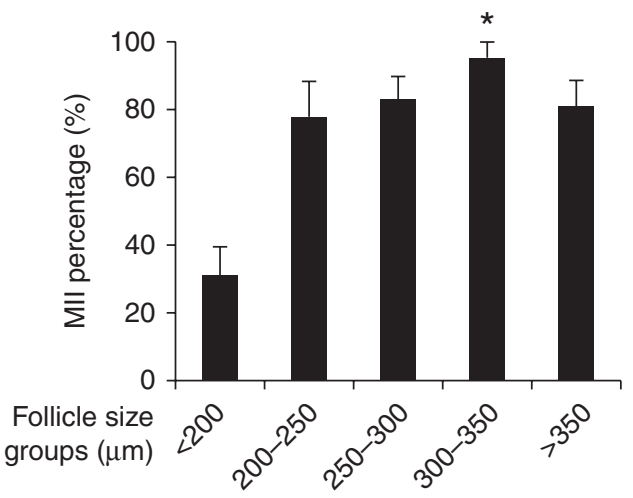

C Follicle diameter with different oocyte maturation outcomes

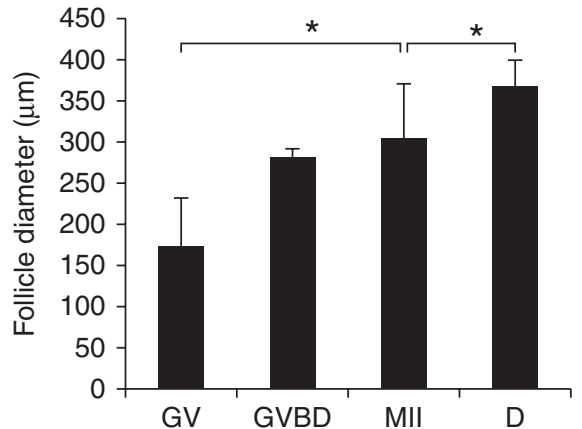

Figure $3 \mathrm{MIl}$ percentages after oocyte maturation for elVFG cultured follicles. (A) MII percentages for follicles cultured for specific periods of time from days 2 to 10. (B) MII percentages for follicles cultured to specific diameters. (C) Follicle diameter associated with different oocyte maturation outcomes. MII, metaphase II; GV, germinal vesicle; GVBD, GV breakdown. D, Degenerated oocyte. ${ }^{*} P<0.05$, compared to the other groups in $\mathrm{A}$ and $\mathrm{B}$, and compared to the diameter of follicles with oocyte in MII stage after oocyte maturation in C; $n=100-160$ follicles for five replicates.

\section{Follicle size is associated with IVF and embryo development outcomes}

To determine the developmental competence of oocytes from different size follicles, MII oocytes from follicles cultured to various diameter ranges were selected for IVF and subsequent monitoring of fertilization and preimplantation embryo development. Eighty-five percent of oocytes from follicles cultured to 300-350 $\mu \mathrm{m}$ reached the $2 \mathrm{PN}$ stage after IVF, $81 \%$ developed to the two-cell embryo stage and $38 \%$ developed to the blastocyst stage. This developmental progression was significantly better than that observed with oocytes from follicles cultured to diameters of $<250,250-300$ or $>350 \mu \mathrm{m}$ (Fig. 5). There was no significant difference in the fertilization percentages or the percentage of twocell embryos for MII oocytes from 300 to $350 \mu \mathrm{m}$ cultured follicles compared to in vivo controls, in which superovulated MII oocytes were used for IVF (Fig. 5). However, the percentage of development to the blastocyst stage was significantly lower for MII oocytes from elVFG compared to MII oocytes derived from in vivo superovulation (Fig. 5). Taken together, these results indicate that, while elVFG may not be able to produce gametes with as high-quality as those follicles produced in vivo, growing follicles to precise terminal diameters of 300-350 $\mu \mathrm{m}$ greatly improves the developmental competence of the resulting gametes.

\section{Discussion}

With the ultimate goal of translating eIVFG to young females with any fertility-threatening condition, tremendous advances have been made in supporting follicle growth and oocyte development in large mammalian species such as domestic dogs, baboons, rhesus monkeys and humans (Xu et al. 2009a,b, 2010, 2011a,b, Songsasen et al. 2011). Rhesus monkey secondary follicles have been cultured in vitro, produced MII oocytes and cleaved to two-cell stage embryos and arrested with uneven cleavage after IVF (Xu et al. 2011a). Previous reports showed that in vivo, the size of isolated follicles correlated with oocyte quality in bovine and porcine models (Lucas et al. 2002, Raghu et al. 2002, Krisher 2004). The goal of this study was to definitively determine the in vitro size index that was most highly correlated to a high-quality gamete, and to then adjust all future studies to size endpoints rather than days in culture, and monitor the follicle individually and choose follicle size as a non-invasive maker to predict follicle maturity.

Here, we demonstrate that oocytes obtain full meiotic and developmental competence when follicle diameters reach $300-350 \mu \mathrm{m}(325 \pm 27 \mu \mathrm{m})$. Although $85 \%$ of oocytes extruded the first polar body when oocyte maturation was performed on day 6 (Fig. 3A), the follicle diameter range on day $6(270-310 \mu \mathrm{m})$ is smaller than the follicles that contained oocytes with the best meiotic and developmental outcomes $(300-350 \mu \mathrm{m})$. The majority $(>60 \%)$ of follicles with a diameter of 300-350 $\mu \mathrm{m}$ were obtained following 7 days of culture, whereas only $19 \%$ were obtained after 8 days, which is the standard culture period for secondary follicles $(\mathrm{Xu}$ et al. 2006a). This reduced meiotic and developmental competence observed following a total of 8 days of 

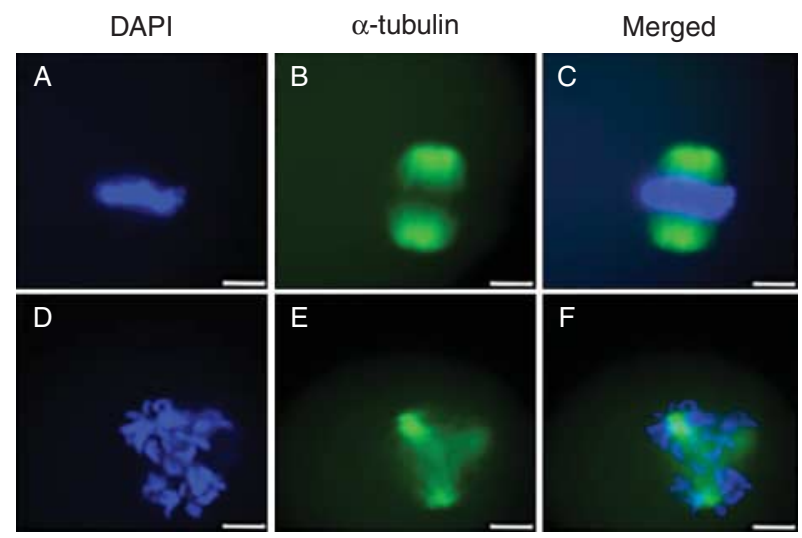

$10 \mu \mathrm{m}$

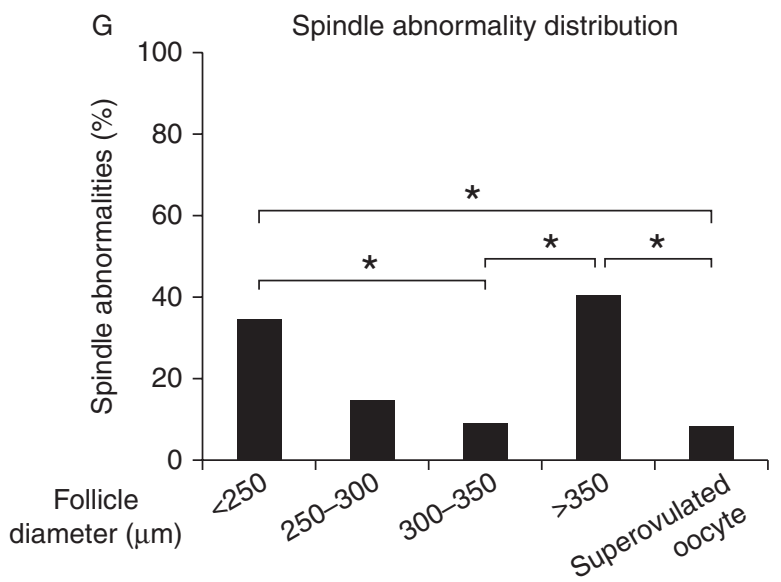

Figure 4 Spindle morphology and chromosome alignment of MII oocytes after oocyte maturation. (A, B and C) Representative images of meiotic spindles of MII oocytes with well-organized microtubule fibers (green) and tightly aligned chromosomes (blue). (D, E and F) Representative images of MII oocytes with abnormal spindle morphology and/or chromosomal alignment. (G) Incidence of spindle abnormality and chromosome misalignment in MII oocytes from follicles cultured to different sizes. MII, metaphase II. ${ }^{*} P<0.05$; $n=20-40$ follicles for three replicates.

culture is also consistent with our findings that these oocytes have a significant increase in unpaired sister chromatids (Supplementary Figure 1, see section on supplementary data given at the end of this article) and are slightly overgrown (Carabatsos et al. 1998, Hutt \& Albertini 2007, Miao et al. 2009). These phenotypes are typically associated with gamete aging, so we propose that prolonged eIVFG results in a new form of aging pre-ovulatory aging - which could account for compromised developmental competence (Tarin et al. 1999, Fissore et al. 2002, Mailhes 2008). These results highlight the need to shift towards size-specific follicle selection for oocyte maturation, which should increase the fraction of high-quality oocytes and improve reproductive outcomes.

A hallmark of oogenesis is the conversion of a highly transcriptionally active growing oocyte into a transcriptionally quiescent oocyte towards the end of its growth phase. This transition strongly correlates with a transition in the oocyte chromatin configuration transition from a NSN to SN state (Watson 2007). Oocytes synthesize and accumulate maternal RNAs to ensure the acquisition of oocyte nuclear and cytoplasmic maturation during oogenesis (Moore \& Lintern-Moore 1978, Eichenlaub-Ritter \& Peschke 2002). It has been reported that the transcriptome of oocytes from follicles cultured in alginate hydrogels is similar to the transcriptome of oocytes developing in vivo, suggesting that eIVFG supports and phenocopies the oocyte development in vitro (Mainigi et al. 2011). Pre-antral follicles, which contain oocytes in a transcriptionally active state, are not meiotically competent, and upon the follicle antral cavity formation and oocyte transcription repression, the oocytes obtain meiotic competence (Zuccotti et al. 1998, Bouniol-Baly et al. 1999). Consistent with previous results in vivo, our data suggest that the oocyte transcription transitions from an active to a quiescent state and oocytes become meiotic-competent when their corresponding follicles develop to the antral stage during elVFG (Figs 1 and 2).
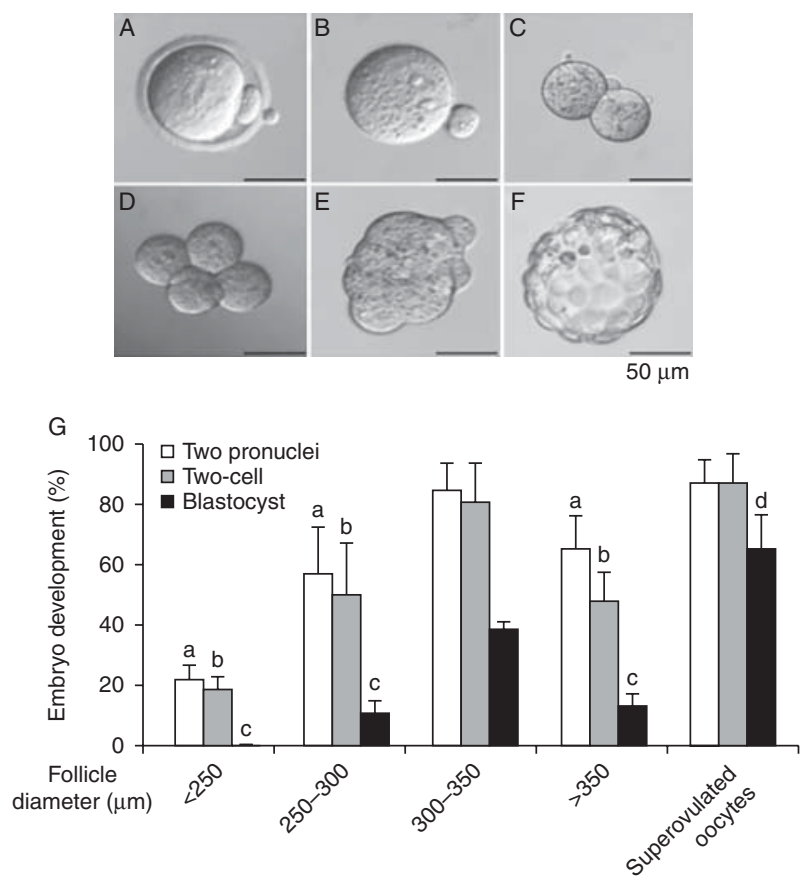

Figure 5 Follicle size is associated with IVF and preimplantation embryo development outcomes. Representative images of MII oocytes after IVF (A) showing two pronuclei (2PN) (B). Fertilized oocyte developed to two-cell (C), four-cell (D), morula (E) and blastocyst stages (F). (G) Developmental outcomes for oocytes from follicles cultured up to specific follicle sizes or from in vivo control superovulated oocytes. a, b and c: $P<0.05$ compared to the percentage of corresponding embryo development stage with follicle size group of $300-350 \mu \mathrm{m}$ and the group of superovulated oocytes; $\mathrm{d}: P<0.05$ between follicle size group of 300-350 $\mu \mathrm{m}$ and superovulated oocytes. Scale bar: $50 \mu \mathrm{m} ; n=30-60$ for three replicates. 
The maturation rate of follicles cultured for a total of 8 days $(80 \%)$ in our study is higher than that in previous reports from our group (63\%) (Xu et al. 2006c). Two factors could have resulted in this higher MII percentage independently of follicle size-based selection methods. First, we used $0.25 \%$ of alginate instead of the $1.5 \%$, which is less rigid, thereby providing a more permissive environment as follicles increase in size to the antral stage. The decreased alginate concentration also promotes the transport and exchange of macromolecules from the culture media or secreted by the follicles, which may promote oocyte development (Xu et al. 2006b). Second, we used a combined growth medium of $\alpha$-MEM and F-12 instead of $\alpha$-MEM medium alone. It has been reported that this combined growth medium improves oocyte meiotic competence (Tagler et al. 2013). F-12 contains various inorganic salts and other components not present in the $\alpha$-MEM such as zinc sulfate and hypoxanthine, which are critical for the oocyte maturation (Eppig et al. 1985, Kim et al. 2010). However, even though our follicle culture strategies in this study are more advanced and result in higher follicle maturation rate, the size-specific follicle selection at a certain follicle diameter range for oocyte maturation significantly improves the oocyte meiotic outcomes compared to absolute follicle culture time.

The percentage of oocytes with an extruded first polar body after oocyte maturation is typically used as a readout of oocyte meiotic competence. However, polar body extrusion is not a sufficient indicator of oocyte quality. In germ cells, the meiotic spindle is a unique subcellular structure composed of microtubules, which is essential for mediating proper chromosome segregation and producing a haploid gamete (Li et al. 2006). In our study, the majority of MII oocytes with normal spindle morphology and chromosome alignment came from follicles with diameters of 300-350 $\mu \mathrm{m}$. However, follicles with diameters $<250$ or $>350 \mu \mathrm{m}$ not only had a lower MII percentage, but the MII oocytes also had a higher proportion of spindle abnormalities and the incidence of chromosome misalignment (Fig. 4), suggesting that both immature and prolonged cultured follicles have compromised oocyte quality.

Follicle survival rate and MII percentage have been used as endpoints in the development of eIVFG conditions (Tagler et al. 2012, 2013); however, markers of developmental competence are equally critical to evaluate oocyte quality. Our data demonstrate that follicles with diameters of 300-350 $\mu \mathrm{m}$ have the highest MII percentage and the best oocyte developmental outcomes. MII oocytes retrieved from follicles ranging between 250 and $300 \mu \mathrm{m}$ in diameter have similar spindle abnormalities as the oocytes from follicles with diameters of $300-350 \mu \mathrm{m}$ and those obtained from superovulation in vivo. Therefore, these oocytes are meiotically competent. However, the fertilization capacity and embryo development outcomes of these oocytes are significantly compromised (Fig. 5), suggesting that the oocyte developmental competence has not yet been acquired, even though meiotic competence has. Repression of oocyte transcription and antral cavity formation correspond to oocyte meiotic competence acquisition (Segers et al. 2010). However, follicle development to this stage $(250-300 \mu \mathrm{m})$ is not sufficient to support full oocyte developmental competence (Fig. 5), suggesting that post-transcriptional activities such as selective transcript degradation, mRNA polyadenylation, and translation are also required at later stages of oocyte development (de Moor \& Richter 2001, Eichenlaub-Ritter \& Peschke 2002, Chen et al. 2011). On the other hand, extending the culture time for follicles to reach diameters $>350 \mu \mathrm{m}$ also had detrimental outcomes. For example, the largest diameter follicles not only produced poor quality oocytes with lower MII percentages and increased oocyte degeneration (Fig. 3), but also contained oocytes with decreased developmental outcomes (Fig. 5), which could be attributed to increased spindle and chromosomal abnormalities (Fig. 4).

In conclusion, our study demonstrates that size-specific follicle selection, rather than absolute culture time, results in the identification of oocytes that will have the best meiotic and developmental outcomes. Oocytes from follicles that lie outside the 300-350 $\mu \mathrm{m}$ size window are immature or have undergone extended culture, both of which compromises gamete quality. Through individualized follicle monitoring, we hope to better identify the optimal endpoint for our elVFG system that best recapitulates in vivo folliculogenesis and produces high-quality oocytes for maturation. The human follicle is one of the most difficult tissues to maintain in culture over extended periods of time. This is likely due to the source of the tissue (generally from an individual with a cancer diagnosis), age, previous treatments and tissue heterogeneity (Picton et al. 2003). The scarcity of the tissue for research (which is expected) and the fact that many follicles do not track from primordial to primary through antral and ovulated follicle. Thus, there are many complexities when growing follicles in culture. That said, we have had success with the mouse, goat, dog, bovine, baboon and rhesus monkey (Xu et al. 2006a, 2009a,b, 2010, 2011a, Songsasen et al. 2011, Silva et al. 2015) and we, and others continue to develop in vivo and in vitro techniques to advance the field (De Vos et al. 2014). We believe the discoveries described in the present report will further refine the elVFG system advancing the clinical application of human follicle culture for women who wish to preserve their fertility in the face of diseases or treatments that may compromise their reproductive health.

\section{Supplementary data}

This is linked to the online version of the paper at http://dx.doi. org/10.1530/REP-15-0175. 


\section{Declaration of interest}

The authors declare that there is no conflict of interest that could be perceived as prejudicing the impartiality of the research reported.

\section{Funding}

This work was supported by National Institutes of Health (NIH) (P50HD076188) to T K Woodruff.

\section{Acknowledgements}

We thank Megan M Romero and Keisha M Barreto (Northwestern University Ovarian Histology: P01HD021921) for sectioning our follicle samples, Rachel M Smith for assistance on experiment design and Stacey C Tobin for editorial assistance on the manuscript.

\section{References}

Bouniol-Baly C, Hamraoui L, Guibert J, Beaujean N, Szollosi MS \& Debey P 1999 Differential transcriptional activity associated with chromatin configuration in fully grown mouse germinal vesicle oocytes. Biology of Reproduction 60 580-587. (doi:10.1095/biolreprod60.3.580)

Carabatsos MJ, Elvin J, Matzuk MM \& Albertini DF 1998 Characterization of oocyte and follicle development in growth differentiation factor-9deficient mice. Developmental Biology 204 373-384. (doi:10.1006/ dbio.1998.9087)

Chen J, Melton C, Suh N, Oh JS, Horner K, Xie F, Sette C, Blelloch R \& Conti M 2011 Genome-wide analysis of translation reveals a critical role for deleted in azoospermia-like (Dazl) at the oocyte-to-zygote transition. Genes and Development 25 755-766. (doi:10.1101/gad. 2028911)

De La Fuente R \& Eppig J 2001 Transcriptional activity of the mouse oocyte genome: companion granulosa cells modulate transcription and chromatin remodeling. Developmental Biology 229 224-236. (doi:10. 1006/dbio.2000.9947)

De Vos M, Smitz J \& Woodruff TK 2014 Fertility preservation in women with cancer. Lancet 384 1302-1310. (doi:10.1016/S0140-6736(14)60834-5)

Duncan FE, Chiang T, Schultz RM \& Lampson MA 2009 Evidence that a defective spindle assembly checkpoint is not the primary cause of maternal age-associated aneuploidy in mouse eggs. Biology of Reproduction 81 768-776. (doi:10.1095/biolreprod.109.077909)

Eichenlaub-Ritter U \& Peschke M 2002 Expression in in-vivo and in-vitro growing and maturing oocytes: focus on regulation of expression at the translational level. Human Reproduction Update 8 21-41. (doi:10.1093/ humupd/8.1.21)

Eppig JJ, Ward-Bailey PF \& Coleman DL 1985 Hypoxanthine and adenosine in murine ovarian follicular fluid: concentrations and activity in maintaining oocyte meiotic arrest. Biology of Reproduction 33 1041-1049. (doi:10.1095/biolreprod33.5.1041)

Evans JP, Schultz RM \& Kopf GS 1995 Mouse sperm-egg plasma membrane interactions: analysis of roles of egg integrins and the mouse sperm homologue of PH-30 (fertilin) beta. Journal of Cell Science 108 3267-3278.

Fissore RA, Kurokawa M, Knott J, Zhang M \& Smyth J 2002 Mechanisms underlying oocyte activation and postovulatory ageing. Reproduction 124 745-754. (doi:10.1530/rep.0.1240745)

Hirshfeld-Cytron J, Gracia C \& Woodruff TK 2011 Nonmalignant diseases and treatments associated with primary ovarian failure: an expanded role for fertility preservation. Journal of Women's Health 20 1467-1477. (doi:10.1089/jwh.2010.2625)

Hutt KJ \& Albertini DF 2007 An oocentric view of folliculogenesis and embryogenesis. Reproductive Biomedicine Online 14 758-764. (doi:10. 1016/S1472-6483(10)60679-7)
Jeruss JS \& Woodruff TK 2009 Preservation of fertility in patients with cancer. New England Journal of Medicine 360 902-911. (doi:10.1056/ NEJMra0801454)

Kim AM, Vogt S, O'Halloran TV \& Woodruff TK 2010 Zinc availability regulates exit from meiosis in maturing mammalian oocytes. Nature Chemical Biology 6 674-681. (doi:10.1038/nchembio.419)

Kito S, Hayao T, Noguchi-Kawasaki Y, Ohta Y, Uhara H \& Tateno S 2004 Improved in vitro fertilization and development by use of modified human tubal fluid and applicability of pronucleate embryos for cryopreservation by rapid freezing in inbred mice. Comparative Medicine 54 564-570.

Krisher RL 2004 The effect of oocyte quality on development. Journal of Animal Science 82 (E-Suppl) E14-E23.

Li Y, Feng HL, Cao YJ, Zheng GJ, Yang Y, Mullen S, Critser JK \& Chen ZJ 2006 Confocal microscopic analysis of the spindle and chromosome configurations of human oocytes matured in vitro. Fertility and Sterility 85 827-832. (doi:10.1016/j.fertnstert.2005.06.064)

Li M, Zhao HC, Li R, Yu Y \& Qiao J 2014 Chromosomal aberrations in in-vitro matured oocytes influence implantation and ongoing pregnancy rates in a mouse model undergoing intracytoplasmic sperm injection. PLOS ONE 9 e103347. (doi:10.1371/journal.pone.0103347)

Lucas X, Martinez EA, Roca J, Vazquez JM, Gil MA, Pastor LM \& Alabart JL 2002 Relationship between antral follicle size, oocyte diameters and nuclear maturation of immature oocytes in pigs. Theriogenology $\mathbf{5 8}$ 871-885. (doi:10.1016/S0093-691X(02)00699-4)

Mailhes JB 2008 Faulty spindle checkpoint and cohesion protein activities predispose oocytes to premature chromosome separation and aneuploidy. Environmental and Molecular Mutagenesis 49 642-658. (doi:10. 1002/em.20412)

Mainigi MA, Ord T \& Schultz RM 2011 Meiotic and developmental competence in mice are compromised following follicle development in vitro using an alginate-based culture system. Biology of Reproduction 85 269-276. (doi:10.1095/biolreprod.111.091124)

McAvey BA, Wortzman GB, Williams CJ \& Evans JP 2002 Involvement of calcium signaling and the actin cytoskeleton in the membrane block to polyspermy in mouse eggs. Biology of Reproduction 67 1342-1352. (doi:10.1095/biolreprod67.4.1342)

Miao YL, Kikuchi K, Sun QY \& Schatten H 2009 Oocyte aging: cellular and molecular changes, developmental potential and reversal possibility. Human Reproduction Update 15 573-585. (doi:10.1093/humupd/ dmp014)

Moon JH, Hyun CS, Lee SW, Son WY, Yoon SH \& Lim JH 2003 Visualization of the metaphase II meiotic spindle in living human oocytes using the Polscope enables the prediction of embryonic developmental competence after ICSI. Human Reproduction 18 817-820. (doi:10.1093/ humrep/deg165)

de Moor CH \& Richter JD 2001 Translational control in vertebrate development. International Review of Cytology 203 567-608. (doi:10. 1016/s0074-7696(01)03017-0)

Moore GP \& Lintern-Moore S 1978 Transcription of the mouse oocyte genome. Biology of Reproduction 18 865-870. (doi:10.1095/biolreprod18.5.865)

Picton HM, Danfour MA, Harris SE, Chambers EL \& Huntriss J 2003 Growth and maturation of oocytes in vitro. Reproduction Supplement 61 $445-462$.

Purcell SH \& Moley KH 2011 The impact of obesity on egg quality. Journal of Assisted Reproduction and Genetics 28 517-524. (doi:10. 1007/s10815-011-9592-y)

Raghu HM, Nandi S \& Reddy SM 2002 Follicle size and oocyte diameter in relation to developmental competence of buffalo oocytes in vitro. Reproduction, Fertility, and Development 14 55-61. (doi:10.1071/ RD01060)

Segers I, Adriaenssens T, Ozturk E \& Smitz J 2010 Acquisition and loss of oocyte meiotic and developmental competence during in vitro antral follicle growth in mouse. Fertility and Sterility 93 2695-2700. (doi:10. 1016/j.fertnstert.2009.11.035)

Silva GM, Rossetto R, Chaves RN, Duarte AB, Araujo VR, Feltrin C, Bernuci MP, Anselmo-Franci JA, Xu M, Woodruff TK et al. 2015 In vitro development of secondary follicles from pre-pubertal and adult goats cultured in two-dimensional or three-dimensional systems. Zygote. In press. (doi:10.1017/s0967199414000070) 
Smitz J, Dolmans MM, Donnez J, Fortune JE, Hovatta O, Jewgenow K, Picton HM, Plancha C, Shea LD, Stouffer RL et al. 2010 Current achievements and future research directions in ovarian tissue culture, in vitro follicle development and transplantation: implications for fertility preservation. Human Reproduction Update 16 395-414. (doi:10.1093/ humupd/dmp056)

Songsasen N, Woodruff TK \& Wildt DE 2011 In vitro growth and steroidogenesis of dog follicles are influenced by the physical and hormonal microenvironment. Reproduction 142 113-122. (doi:10.1530/ REP-10-0442)

Tagler D, Tu T, Smith RM, Anderson NR, Tingen CM, Woodruff TK \& Shea LD 2012 Embryonic fibroblasts enable the culture of primary ovarian follicles within alginate hydrogels. Tissue Engineering. Part A 18 1229-1238. (doi:10.1089/ten.tea.2011.0418)

Tagler D, Makanji Y, Anderson NR, Woodruff TK \& Shea LD 2013 Supplemented $\alpha$ MEM/F12-based medium enables the survival and growth of primary ovarian follicles encapsulated in alginate hydrogels. Biotechnology and Bioengineering 110 3258-3268. (doi:10.1002/bit.24986)

Tarin JJ, Perez-Albala S, Aguilar A, Minarro J, Hermenegildo C \& Cano A 1999 Long-term effects of postovulatory aging of mouse oocytes on offspring: a two-generational study. Biology of Reproduction $\mathbf{6 1}$ 1347-1355. (doi:10.1095/biolreprod61.5.1347)

Telfer EE \& McLaughlin M 2012 Strategies to support human oocyte development in vitro. International Journal of Developmental Biology 56 901-907. (doi:10.1387/ijdb.130001et)

Watson AJ 2007 Oocyte cytoplasmic maturation: a key mediator of oocyte and embryo developmental competence. Journal of Animal Science 85 E1-E3. (doi:10.2527/jas.2006-432)

Woodruff TK 2007 The emergence of a new interdiscipline: oncofertility. Cancer Treatment and Research 138 3-11. (doi:10.1007/978-0-38772293-1_1)

Xu M, Kreeger PK, Shea LD \& Woodruff TK 2006a Tissue-engineered follicles produce live, fertile offspring. Tissue Engineering 12 2739-2746. (doi:10.1089/ten.2006.12.2739)

Xu M, West E, Shea LD \& Woodruff TK $2006 b$ Identification of a stagespecific permissive in vitro culture environment for follicle growth and oocyte development. Biology of Reproduction 75 916-923. (doi:10.1095/biolreprod.106.054833)
Xu M, West E, Shea LD \& Woodruff TK 2006c Identification of a stagespecific permissive in vitro culture environment for follicle growth and oocyte development. Biology of Reproduction 75 916-923. (doi:10.1095/biolreprod.106.054833)

Xu M, Barrett SL, West-Farrell E, Kondapalli LA, Kiesewetter SE, Shea LD \& Woodruff TK 2009a In vitro grown human ovarian follicles from cancer patients support oocyte growth. Human Reproduction 24 2531-2540. (doi:10.1093/humrep/dep228)

Xu M, West-Farrell ER, Stouffer RL, Shea LD, Woodruff TK \& Zelinski MB $2009 \mathrm{~b}$ Encapsulated three-dimensional culture supports development of nonhuman primate secondary follicles. Biology of Reproduction $\mathbf{8 1}$ 587-594. (doi:10.1095/biolreprod.108.074732)

Xu J, Bernuci MP, Lawson MS, Yeoman RR, Fisher TE, Zelinski MB \& Stouffer RL 2010 Survival, growth, and maturation of secondary follicles from prepubertal, young, and older adult rhesus monkeys during encapsulated three-dimensional culture: effects of gonadotropins and insulin. Reproduction 140 685-697. (doi:10.1530/REP-10-0284)

Xu J, Lawson MS, Yeoman RR, Pau KY, Barrett SL, Zelinski MB \& Stouffer RL 2011a Secondary follicle growth and oocyte maturation during encapsulated three-dimensional culture in rhesus monkeys: effects of gonadotrophins, oxygen and fetuin. Human Reproduction 26 1061-1072. (doi:10.1093/humrep/der049)

Xu M, Fazleabas AT, Shikanov A, Jackson E, Barrett SL, Hirshfeld-Cytron J, Kiesewetter SE, Shea LD \& Woodruff TK $2011 b$ In vitro oocyte maturation and preantral follicle culture from the luteal-phase baboon ovary produce mature oocytes. Biology of Reproduction 84 689-697. (doi:10.1095/biolreprod.110.088674)

Zuccotti M, Giorgi Rossi P, Martinez A, Garagna S, Forabosco A \& Redi CA 1998 Meiotic and developmental competence of mouse antral oocytes. Biology of Reproduction 58 700-704. (doi:10.1095/biolreprod58.3.700)

Received 15 April 2015

First decision 26 May 2015

Revised manuscript received 9 June 2015

Accepted 18 June 2015 